\title{
Low Diagnostic Yield of Elective Coronary Angiography
}

\author{
Manesh R. Patel, M.D., Eric D. Peterson, M.D., M.P.H., David Dai, M.S., \\ J. Matthew Brennan, M.D., Rita F. Redberg, M.D., H. Vernon Anderson, M.D., \\ Ralph G. Brindis, M.D., and Pamela S. Douglas, M.D.
}

From the Duke Clinical Research Institute, Duke University, Durham, NC (M.R.P., E.D.P., D.D., J.M.B., P.S.D.); University of California at San Francisco, San Francisco (R.F.R., R.G.B.); and the University of Texas Health Science Center, Houston (H.V.A.). Address reprint requests to Dr. Patel at the Duke Clinical Research Institute, Duke University Medical Center, P.O. Box 17969, Durham, NC 27715, or at manesh.patel@duke.edu.

N Engl J Med 2010;362:886-95.

Copyright (c) 2010 Massachusetts Medical Society.

\section{ABSTRACT}

\section{BACKGROUND}

Guidelines for triaging patients for cardiac catheterization recommend a risk assessment and noninvasive testing. We determined patterns of noninvasive testing and the diagnostic yield of catheterization among patients with suspected coronary artery disease in a contemporary national sample.

\section{METHODS}

From January 2004 through April 2008, at 663 hospitals in the American College of Cardiology National Cardiovascular Data Registry, we identified patients without known coronary artery disease who were undergoing elective catheterization. The patients' demographic characteristics, risk factors, and symptoms and the results of noninvasive testing were correlated with the presence of obstructive coronary artery disease, which was defined as stenosis of $50 \%$ or more of the diameter of the left main coronary artery or stenosis of $70 \%$ or more of the diameter of a major epicardial vessel.

\section{RESULTS}

A total of 398,978 patients were included in the study. The median age was 61 years; $52.7 \%$ of the patients were men, $26.0 \%$ had diabetes, and $69.6 \%$ had hypertension. Noninvasive testing was performed in $83.9 \%$ of the patients. At catheterization, 149,739 patients (37.6\%) had obstructive coronary artery disease. No coronary artery disease (defined as $<20 \%$ stenosis in all vessels) was reported in $39.2 \%$ of the patients. Independent predictors of obstructive coronary artery disease included male sex (odds ratio, 2.70; 95\% confidence interval [CI], 2.64 to 2.76), older age (odds ratio per 5-year increment, 1.29; 95\% CI, 1.28 to 1.30), presence of insulin-dependent diabetes (odds ratio, 2.14; 95\% CI, 2.07 to 2.21), and presence of dyslipidemia (odds ratio, $1.62 ; 95 \% \mathrm{CI}, 1.57$ to 1.67 ). Patients with a positive result on a noninvasive test were moderately more likely to have obstructive coronary artery disease than those who did not undergo any testing ( $41.0 \%$ vs. $35.0 \%$; $\mathrm{P}<0.001$; adjusted odds ratio, 1.28 ; $95 \% \mathrm{CI}, 1.19$ to 1.37 ).

\section{CONCLUSIONS}

In this study, slightly more than one third of patients without known disease who underwent elective cardiac catheterization had obstructive coronary artery disease. Better strategies for risk stratification are needed to inform decisions and to increase the diagnostic yield of cardiac catheterization in routine clinical practice. 
$\mathrm{F}$ OR PATIENTS IN STABLE CONDITION WHO are undergoing assessment for obstructive coronary artery disease, guidelines recommend continued observation in the case of patients who are at very low risk, noninvasive stress testing to determine the need for cardiac catheterization in the case of patients at intermediate risk, and direct referral for catheterization in the case of patients at high risk. ${ }^{1-3}$ The goal of these recommendations is to limit the number of patients without obstructive coronary artery disease who undergo invasive angiography and thereby enhance the diagnostic yield of cardiac catheterization.

The use of both noninvasive and invasive studies has grown substantially ${ }^{4}$ t the Government Accountability Office reported a doubling in the cost of imaging services from 2000 to 2006, with $\$ 14.1$ billion in Medicare spending for imaging services in 2006 alone (www.gao.gov/products/ GAO-08-452). The increased use of noninvasive testing should result in more effective risk stratification of patients, allowing identification of those patients who would be most likely to benefit from cardiac catheterization and ideally reducing the use of this invasive procedure in patients who do not have obstructive disease.

We analyzed data from patients in a contemporary, large, national cardiac-catheterization registry to assess the effectiveness of current practices in enhancing the yield of diagnostic cardiac catheterization as measured by the prevalence of obstructive coronary artery disease. We also examined the associations among clinical risk factors, presenting symptoms, results of noninvasive tests, and results of subsequent catheterization.

METHODS

\section{DATA SOURCES}

The CathPCI Registry of the National Cardiovascular Data Registry (NCDR), which is sponsored by the American College of Cardiology and the Society for Cardiovascular Angiography and Interventions, has been described previously. ${ }^{5,6}$ Briefly, the NCDR CathPCI Registry is a large, voluntary registry of clinical data and in-hospital outcome data associated with diagnostic cardiac catheterization and percutaneous coronary intervention, collected from more than 800 U.S. sites. Information on characteristics of the patients and hospitals, findings of procedures that are performed, interventions that are undertaken, and the outcomes is collected at participating hospitals on the basis of explicit, prespecified data elements defined by an NCDR committee (www.ncdr.com/ webncdr/DefaultCathPCI.aspx). Only institutions whose submissions meet quality criteria for data reporting are included.

\section{STUDY POPULATION}

All patients in the NCDR for whom there were complete data on diagnostic cardiac catheterization for the period from January 2004 through April 2008 were included (Fig. 1). To identify patients without known heart disease, patients with a history of myocardial infarction, percutaneous coronary intervention, coronary-artery bypass surgery, cardiac transplantation, or valvular surgery were excluded, as were patients with indications for emergency or urgent cardiac catheterization (acute coronary syndromes, acute myocardial infarction, or cardiogenic shock) and patients who were being evaluated for transplantation or who were about to undergo valvular surgery. The institutional review board at Duke University Medical Center granted a waiver of written informed consent and gave authorization for this study.

Information was collected on the patients' demographic characteristics, clinical risk factors, and symptoms and on the results of noninvasive tests. Symptoms were categorized as no symptoms (including no angina), atypical chest pain, or stable angina. Noninvasive diagnostic tests included tests that were performed "to rule out ischemia prior to the procedure" - defined in the case-report form as "ECG, exercise or pharmacologic stress tests, radionucleotide, echo, CT scans or other heart scans" (www.ncdr.com/webncdr/DefaultCathPCI .aspx). The results were categorized as positive, negative, or equivocal. A modified Framingham risk score (www.framinghamheartstudy.org/risk/ coronary.html) was calculated on the basis of available clinical data, with a moderate score (i.e., 1 point) imputed for either a history of dyslipidemia or the use of statins and for the presence of hypertension or a history of medication use for the treatment of high blood pressure.

\section{OBSTRUCTIVE CORONARY ARTERY DISEASE}

Obstructive coronary artery disease was defined as stenosis of $50 \%$ or more of the diameter of the left main coronary artery or stenosis of $70 \%$ or more of the diameter of a major epicardial or branch vessel that was more than $2.0 \mathrm{~mm}$ in di- 


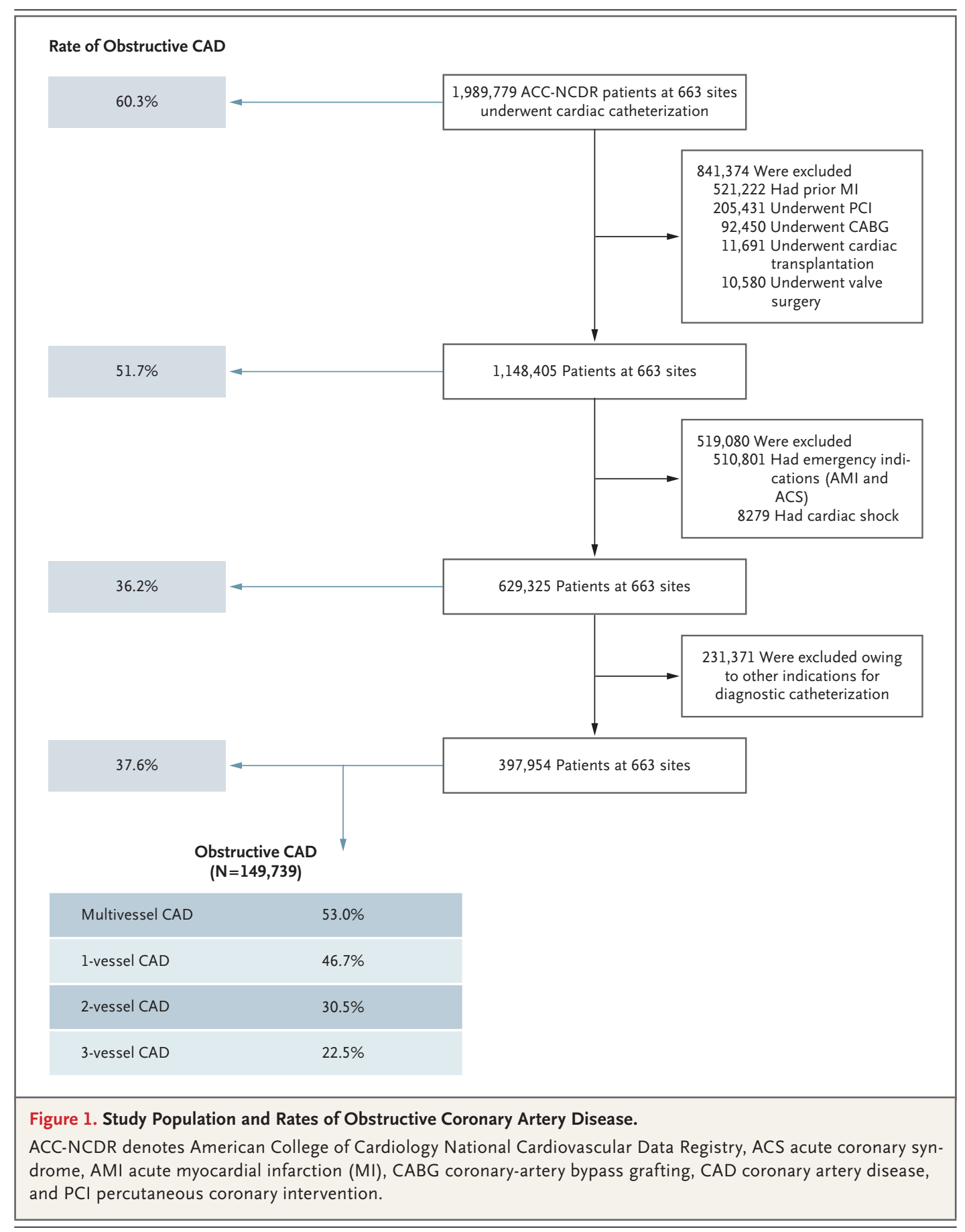

ameter. For a sensitivity analysis, we broadened the definition of obstructive coronary artery disease to include stenosis of $50 \%$ or more in any coronary vessel. Patients with no coronary artery disease, which was defined as stenosis of less than $20 \%$ in all vessels, were also identified. The degree of stenosis was determined by physicians at each site and is defined in the NCDR CathPCI
Registry as the percentage reduction in diameter, as estimated from a comparison with the diameter of the normal reference vessel proximal to the lesion.

\section{STATISTICAL ANALYSIS}

We compared the baseline demographic characteristics, risk factors, symptoms, and results of 
noninvasive tests for patients with obstructive coronary artery disease with those for patients without obstructive coronary artery disease. Continuous variables are presented as medians and interquartile ranges, and categorical variables as percentages. Continuous variables were compared with the use of the Wilcoxon-Mann-Whitney nonparametric test, and categorical variables with the use of the chi-square test. We also assessed temporal trends in the prevalence of obstructive coronary artery disease.

Multivariate logistic-regression analysis was performed to identify factors associated with obstructive coronary artery disease from among candidate baseline clinical variables (Table 1). Generalized estimating equations ${ }^{7}$ were used to

\begin{tabular}{|c|c|c|c|c|}
\hline Characteristic & $\begin{array}{c}\text { Total } \\
(\mathrm{N}=397,954)\end{array}$ & $\begin{array}{c}\text { Obstructive Coronary } \\
\text { Artery Disease } \\
(\mathrm{N}=149,739)\end{array}$ & $\begin{array}{c}\text { No Obstructive Coronary } \\
\text { Artery Disease } \\
(\mathrm{N}=248,215)\end{array}$ & P Value \\
\hline Age (yr) & & & & $<0.001$ \\
\hline Median & 61 & 66 & 58 & \\
\hline Interquartile range & $52-70$ & $58-74$ & $50-68$ & \\
\hline Female sex (\%) & 47.3 & 33.9 & 55.4 & $<0.001$ \\
\hline White race $(\%) *$ & 82.5 & 84.8 & 81.1 & $<0.001$ \\
\hline \multicolumn{5}{|l|}{ Clinical risk factors } \\
\hline Body-mass index & & & & $<0.001$ \\
\hline Median & 29.6 & 28.9 & 30.0 & \\
\hline Interquartile range & $25.9-34.4$ & $25.7-33.1$ & $26.1-35.3$ & \\
\hline Use of tobacco (\%) & & & & $<0.001$ \\
\hline Former & 32.1 & 36.8 & 29.2 & \\
\hline Current & 19.8 & 19.7 & 19.9 & \\
\hline Diabetes (\%) & & & & $<0.001$ \\
\hline Any & 26.0 & 32.0 & 22.4 & \\
\hline Insulin-dependent & 6.4 & 8.4 & 5.2 & $<0.001$ \\
\hline Hypertension (\%) & 69.6 & 76.4 & 65.5 & $<0.001$ \\
\hline Family history of coronary artery disease (\%) & 30.0 & 28.8 & 30.7 & $<0.001$ \\
\hline Dyslipidemia (\%) & 62.5 & 71.8 & 56.8 & $<0.001$ \\
\hline Creatinine clearance $(\mathrm{ml} / \mathrm{min}) ‡$ & & & & $<0.001$ \\
\hline Median & 67.4 & 63.7 & 69.6 & \\
\hline Interquartile range & $51.7-84.6$ & $48.3-80.5$ & $53.9-87.2$ & \\
\hline Peripheral vascular disease (\%) & 7.0 & 10.5 & 4.9 & $<0.001$ \\
\hline Cerebrovascular disease (\%) & 7.1 & 9.6 & 5.5 & $<0.001$ \\
\hline Chronic obstructive pulmonary disease (\%) & 14.4 & 13.5 & 15.0 & $<0.001$ \\
\hline \multicolumn{5}{|l|}{ Framingham risk score (\%) } \\
\hline Low & 29.2 & 13.5 & 38.6 & $<0.001$ \\
\hline Intermediate & 55.0 & 59.4 & 52.4 & \\
\hline High & 15.8 & 27.1 & 13.5 & \\
\hline \multicolumn{5}{|l|}{ Clinical presentation (\%) } \\
\hline No symptoms, including no angina & 30.0 & 31.5 & 29.1 & $<0.001$ \\
\hline Atypical symptoms & 36.8 & 24.6 & 44.2 & $<0.001$ \\
\hline Stable angina & 33.2 & 43.9 & 26.7 & $<0.001$ \\
\hline
\end{tabular}

* Race was determined by the investigator at the site.

$\dagger$ The body-mass index is the weight in kilograms divided by the square of the height in meters.

$\uparrow$ The Modification of Diet in Renal Disease formula was used to estimate the creatinine clearance rate. 
account for within-hospital clustering, since patients at the same hospital may have similar disease profiles. The Wald chi-square test was used to determine significant predictors of obstructive coronary artery disease. To understand the relative value of the factors in predicting obstructive coronary artery disease, we constructed four separate models; we started with a model for predicting risk if no symptoms were present, as assessed with the use of the Framingham risk score alone, and then added into the model other clinical factors, followed by documented symptoms, and finally the results of noninvasive testing. The predictive value of each model is represented by the C-statistic. A nonparametric approach for comparing each C-statistic was used. ${ }^{8} \mathrm{P}$ values of less than 0.05 were considered to indicate statistical significance for all tests. All statistical analyses were performed by the Duke Clinical Research Institute with the use of SAS software, version 9.2 (SAS Institute).

\section{RESULTS}

\section{STUDY POPULATION}

From January 2004 through April 2008, data for $1,989,779$ patients undergoing cardiac catheterization at 663 sites were entered into the NCDR (Fig. 1). We excluded 841,374 of these patients $(42.3 \%)$ because they had a known history of heart disease and 519,080 patients (26.1\%) because they underwent emergency or urgent cardiac catheterization. Our final data set for analysis included 397,954 patients without known coronary artery disease who were undergoing elective cardiac catheterization, a cohort that represented $20.0 \%$ of all patients undergoing catheterization.

\section{PREVALENCE OF OBSTRUCTIVE CORONARY ARTERY DISEASE}

Obstructive coronary artery disease was identified in $37.6 \%$ of the patients, of whom $53.0 \%$ had evidence of multivessel disease (Fig. 1). Of all patients undergoing diagnostic catheterization, 8.5\% had three-vessel disease and 3.9\% had left main coronary artery disease. If the definition of obstructive coronary artery disease was broadened to include stenosis of $50 \%$ or more in any coronary vessel, the prevalence increased to $41.0 \%$. In contrast, $39.2 \%$ of the patients had no coronary artery disease $(<20 \%$ stenosis in all vessels). There was a small but significant increase from 2004 to 2008 in the prevalence of obstructive coronary artery disease (from $36.8 \%$ to $38.8 \%, \mathrm{P}<0.001)$.

\section{BASELINE CHARACTERISTICS}

In general, patients with obstructive coronary artery disease, as compared with patients who did not have obstructive coronary artery disease, were older (median age, 66 vs. 58 years), more likely to be men $(66.1 \%$ vs. $44.6 \%)$, and more likely to have diabetes $(32.0 \%$ vs. $22.4 \%)$, hypertension $(76.4 \%$ vs. $65.5 \%$ ), or dyslipidemia ( $71.8 \%$ vs. $56.8 \%$ ) ( $\mathrm{P}<0.001$ for all comparisons) (Table 1). Patients with symptoms of stable angina were more likely to have obstructive coronary artery disease than were patients without symptoms ( $43.9 \%$ vs. $31.5 \%$, $\mathrm{P}<0.001)$.

\section{NONINVASIVE TESTING}

Noninvasive testing (resting electrocardiography, echocardiography, computed tomography [CT], or a stress test) was performed in $83.9 \%$ of the patients before invasive angiography. A positive test result was recorded in the case of $68.6 \%$ of all the patients in the cohort. A noninvasive test was not performed before angiography in $17.1 \%$ of lowrisk patients, $15.9 \%$ of intermediate-risk patients, and $15.0 \%$ of high-risk patients $(\mathrm{P}<0.001)$.

Patients with a positive result on a noninvasive test had a slightly higher rate of obstructive coronary artery disease than did patients who did not undergo any noninvasive testing before angiography $(41.0 \%$ vs. $35.0 \%, \mathrm{P}<0.001)$; the rate of obstructive coronary artery disease among patients with a positive test result was also higher than the rate among those with equivocal test results and those with negative test results (41.3\%, vs. $27.1 \%$ and $28.3 \%$, respectively). Since the data set included asymptomatic and symptomatic patients, the association between rates of obstructive coronary artery disease and the results of noninvasive tests are presented in Figure 2 according to Framingham risk-score categories (low, intermediate, or high) and symptom categories (no symptoms, atypical symptoms, or angina). The diagnostic yield for obstructive coronary artery disease increased with a higher Framingham risk score, as well as with the presence of angina ( $\mathrm{P}<0.001$ for both analyses). 


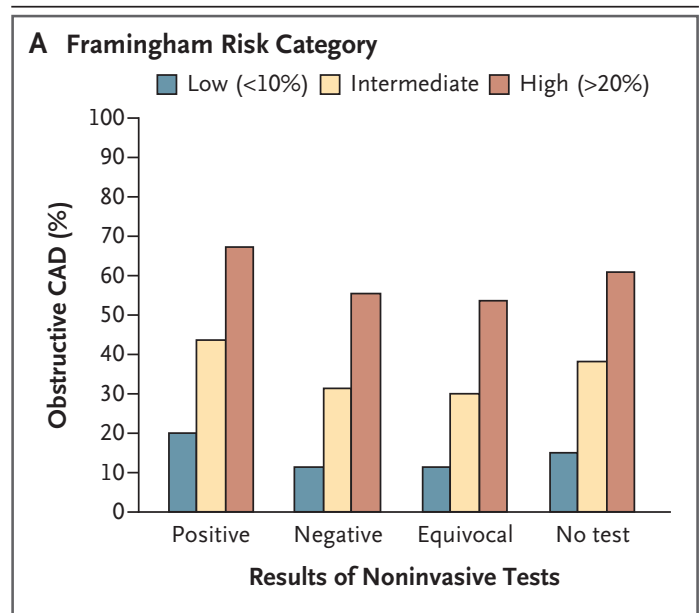

B Symptom Characteristic

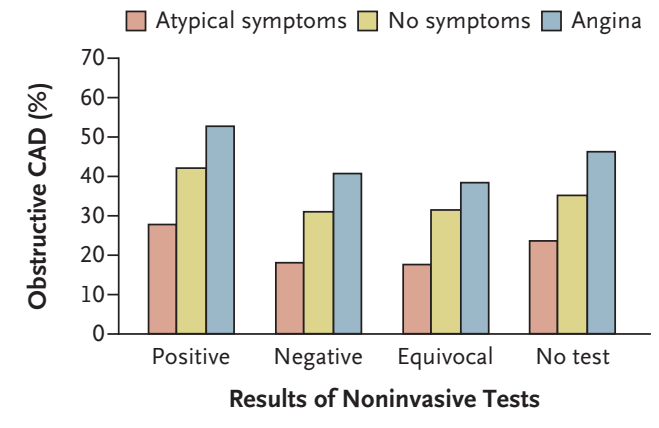

Figure 2. Patients with Obstructive Coronary Artery Disease, According to Noninvasive Test Result.

Results are presented according to the level of the Framingham risk score (low, intermediate, or high) (Panel A) and symptom category (no symptoms, atypical symptoms, or angina) (Panel B). CAD denotes coronary artery disease.

\section{PREDICTORS OF OBSTRUCTIVE CORONARY ARTERY DISEASE}

Male sex, white race, older age, lower body-mass index, use of tobacco, and presence of diabetes, dyslipidemia, peripheral vascular disease, cerebrovascular disease, dialysis-dependent renal failure, and hypertension were each independent predictors of obstructive coronary artery disease (Table 2). A positive result on a noninvasive test, as compared with no testing, was independently associated with obstructive coronary artery disease (adjusted odds ratio, 1.28; 95\% confidence interval [CI], 1.19 to 1.37), but to a lesser extent than were major clinical risk factors. As expected, typical angina was associated with obstructive disease (odds ratio, 1.91; 95\% CI, 1.78 to 2.05). There was no association between hospital characteristics and obstructive disease (data not shown).

\section{INCREMENTAL VALUE OF INFORMATION OBTAINED BEFORE ANGIOGRAPHY}

The results of four separate models for the prediction of obstructive coronary artery disease with the use of information obtained before invasive angiography was performed are presented in Figure 3. The first model included only a modified Framingham risk score (C-statistic, 0.670; 95\% CI, 0.669 to 0.672 ). In the second model, clinical factors that were not included in the Framingham risk score were added, such as the body-mass index; presence or absence of peripheral arterial disease, cerebrovascular disease, and obstructive lung disease; and need or no need for dialysis. The C-statistic for this model was 0.742 (95\% CI, 0.740 to 0.743 ). A slight increase in the model's predictive ability was noted with the addition in the third model of symptom characteristics for the overall population (C-statistic, $0.761 ; 95 \% \mathrm{CI}$, 0.759 to 0.762 ), with a similar effect across Framingham risk levels. Finally, although a positive noninvasive test was associated with the presence of obstructive coronary artery disease, the addition of information obtained from noninvasive tests (model 4) had a limited effect on the model's predictive ability over and above the effect achieved from the addition of clinical risk factors and symptoms (C-statistic, 0.764; 95\% CI, 0.762 to 0.765 ).

\section{DISCUSSION}

In this large, representative sample of patients without known coronary artery disease who underwent invasive angiography in the United States during the period from January 2004 through April 2008, cardiac catheterization had a low diagnostic yield. A minority of patients undergoing this invasive test (37.6\%) had obstructive coronary artery disease (i.e., $\geq 50 \%$ stenosis of the left main coronary artery or $\geq 70 \%$ stenosis of a major epicardial vessel). The percentage was similar $(41.0 \%)$ when the definition of obstructive disease was expanded to include stenosis of $50 \%$ or more of any coronary vessel. We also found that, although certain demographic and clinical characteristics could be useful in determining the likelihood that obstructive coronary artery disease 


\begin{tabular}{|c|c|c|}
\hline Variable & $\begin{array}{l}\text { Wald Chi-Square } \\
\text { Statistic }\end{array}$ & $\begin{array}{c}\text { Adjusted Odds Ratio } \\
(95 \% \mathrm{Cl})\end{array}$ \\
\hline Age, per 5-yr increase & 6146.2 & $1.29(1.28-1.30)$ \\
\hline $\begin{array}{l}\text { Body-mass index, per 5-unit } \\
\text { increase* }\end{array}$ & 550.3 & $0.92(0.91-0.92)$ \\
\hline Male sex & 8632.9 & $2.70(2.64-2.76)$ \\
\hline White race ${ }^{\dagger}$ & 50.2 & $1.21(1.15-1.28)$ \\
\hline \multicolumn{3}{|l|}{ Diabetest } \\
\hline Insulin-dependent & 1932.3 & $2.14(2.07-2.21)$ \\
\hline Non-insulin-dependent & 1187.8 & $1.45(1.42-1.48)$ \\
\hline Dyslipidemia & 972.8 & $1.62(1.57-1.67)$ \\
\hline \multicolumn{3}{|l|}{ Use of tobacco } \\
\hline Current & 790.4 & $1.50(1.45-1.54)$ \\
\hline Former & 34.3 & $1.09(1.06-1.12)$ \\
\hline Hypertension & 561.4 & $1.29(1.26-1.32)$ \\
\hline Peripheral vascular disease & 449.5 & $1.54(1.48-1.61)$ \\
\hline Cerebrovascular disease & 197.8 & $1.26(1.21-1.30)$ \\
\hline Ejection fraction, per $5 \%$ increase & 374.7 & $1.08(1.07-1.09)$ \\
\hline Congestive heart failure & 83.1 & $0.80(0.76-0.84)$ \\
\hline $\begin{array}{c}\text { Glomerular filtration rate, per } \\
\text { 5-unit increase } \mathbb{\int}\end{array}$ & 31.3 & $1.01(1.00-1.01)$ \\
\hline \multicolumn{3}{|l|}{ Renal failure } \\
\hline Requiring dialysis & 26.9 & $1.30(1.18-1.43)$ \\
\hline Not requiring dialysis & 14.1 & $1.45(1.07-1.23)$ \\
\hline Chronic lung disease & 298.6 & $0.78(0.76-0.80)$ \\
\hline \multicolumn{3}{|l|}{ Presence of symptoms\| } \\
\hline Typical & 353.6 & $1.91(1.78-2.05)$ \\
\hline Atypical & 84.2 & $0.76(0.71-0.80)$ \\
\hline \multicolumn{3}{|l|}{ Noninvasive testing** } \\
\hline Positive result & 48.9 & $1.28(1.19-1.37)$ \\
\hline Equivocal result & 25.3 & $0.79(0.71-0.86)$ \\
\hline Negative result & 19.4 & $0.82(0.74-0.89)$ \\
\hline
\end{tabular}

* The body-mass index is the weight in kilograms divided by the square of the height in meters.

$\dagger$ Race was determined by the investigator at the site.

$\neq$ The odds ratios are for insulin-dependent diabetes and non-insulin-dependent diabetes as compared with no diabetes.

$\int$ The Modification of Diet in Renal Disease formula was used to estimate the glomerular filtration rate.

q The odds ratios are for renal failure requiring dialysis and renal failure not requiring dialysis as compared with no renal failure.

| The odds ratios are for typical symptoms and atypical symptoms as compared with no symptoms.

$* *$ The odds ratios are for positive, equivocal, and negative results of noninvasive tests as compared with no noninvasive testing.

would be present, the incremental value of a positive result on a noninvasive test (including any of a broad range of tests such as resting electrocardiography, echocardiography, CT, or stress test) was limited.
Although the rate at which coronary arteries are found to be "normal" among patients who have undergone invasive angiography has been discussed as a potential performance measure, ${ }^{9}$ rates that have been published previously have important limitations. In a 1992 report by the RAND Corporation, between 9 and 36\% of patients who underwent invasive angiography were found to have normal coronary arteries, but the findings were limited by varying definitions of normal and by varying radiographic systems. ${ }^{10}$ Findings from the Coronary Artery Surgery Study (CASS), involving 21,487 angiograms, showed that $18.8 \%$ of patients had nonobstructive coronary artery disease, which was defined as stenosis of less than $50 \%$ in all vessels. ${ }^{11}$ Data from the Society for Cardiac Angiography and Interventions (SCAI) Registry, which are somewhat more recent than the CASS findings but are still more than 15 years old, showed that between 20 and $27 \%$ of patients had normal coronary arteries and between 30 and $35 \%$ of patients had stenosis of less than $50 \%$ in all vessels. ${ }^{12,13}$ Although these data were derived from more than 200,000 patients over the course of several years, they were obtained before the current increase in both noninvasive imaging and the use of cardiac catheterization and reflect a broad spectrum of patients undergoing angiography, including those who were being evaluated for acute myocardial infarction and other emergency or urgent indications.

The low rate of obstructive coronary artery disease in the current NCDR analysis may reflect the stricter inclusion criteria for the patient population under study. Our study excluded patients with a known history of coronary artery disease, those undergoing emergency or urgent angiography in the setting of acute myocardial infarction or clinical instability, and those undergoing elective angiography before transplantation or valvular surgery. When data from the entire NCDR population were analyzed irrespective of prior disease or clinical indication, the rate of obstructive coronary artery disease increased to $60.3 \%$ (Fig. 1), which is similar to the rates in previous reports. ${ }^{14}$

Thus, the definition of the patient population is critical in placing our findings in context. We believe that our inclusion of a narrower population than that used in previous studies is helpful for understanding and eventually improving the clinical decision-making process that leads to the diagnostic use of cardiac catheterization. 


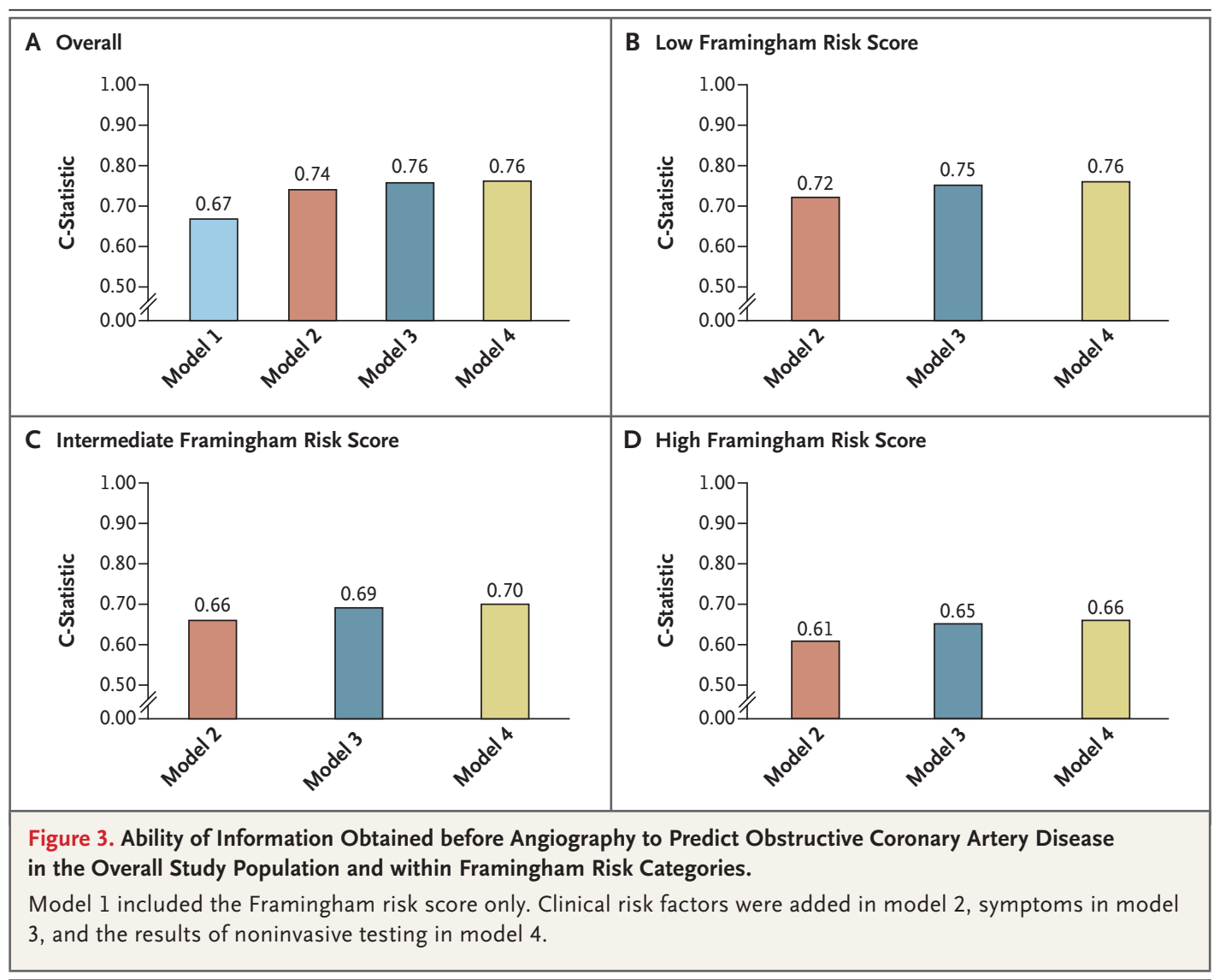

To better understand the relationship between current decision-making processes regarding the need for invasive angiography and its diagnostic yield, we explored the usefulness of known major cardiac risk factors, symptoms, and noninvasive testing results for predicting the presence of obstructive coronary artery disease. Not surprisingly, the strongest independent predictive factors were the traditional risk factors, including older age, male sex, use of tobacco, and the presence of diabetes, dyslipidemia, or hypertension. In addition to consideration of clinical risk factors, the probability of obstructive coronary artery disease, as assessed before angiography, is generally estimated by considering the presence or absence of symptoms, on the basis of work by Diamond and Forrester, ${ }^{15}$ which was validated in independent clinical data sets. ${ }^{16}$ When data on symptoms were added to our predictive model, they were independently related to the presence of obstructive coronary artery disease and also slightly improved the predictive ability of the model for obstructive coronary artery disease (from a C-statistic of 0.7417 to a C-statistic of 0.7609 ).
This study also provides insights into patterns of noninvasive testing among patients undergoing diagnostic cardiac catheterization. Data from Lin et al. showed that in a Medicare population undergoing elective percutaneous coronary intervention, only $41 \%$ of the patients underwent stress testing. ${ }^{17}$ In contrast, our study showed that up to $84 \%$ of patients undergoing cardiac catheterization had undergone a previous noninvasive diagnostic test. This difference probably reflects the fact that NCDR includes a broad range of tests that are classified as noninvasive, whereas the analysis in the article by Lin et al. included only tests for ischemia. We found that noninvasive testing was paradoxically used more often in patients with a high Framingham risk score than in those with an intermediate or low risk score, a practice that is at odds with current guideline recommendations. ${ }^{3}$

We also found that a positive noninvasive test was independently related to the presence of obstructive coronary artery disease. Although the association was significant, the effect of a positive noninvasive test on the ability of the model to 
predict the presence of obstructive coronary artery disease was limited, increasing the $\mathrm{C}$-statistic from 0.7609 to 0.7639 . However, we cannot evaluate the performance of noninvasive testing, because we have no information on patients who underwent noninvasive testing but were not referred for catheterization, a situation that was probably more common among patients with negative or equivocal test results. In addition, the specific noninvasive test that was used in the NCDR is unknown and could have been one of a broad range of tests. Although the NCDR casereport form was intended to identify noninvasive tests for ischemia, resting electrocardiography, echocardiography, or CT may have been included. Finally, if testing is used extensively in low-risk patient populations, Bayesian principles dictate that many positive test results will be false positive results rather than true positive results, and this may have severely limited the ability of noninvasive testing to add incremental value for the identification of obstructive coronary artery disease.

Our results suggest that greater focus should be placed on the $30.0 \%$ of patients who were noted to have no symptoms, including no angina. Presumably the decision to proceed with invasive catheterization in the case of these patients was driven by clinical assessment of risk, testing for ischemia, or both. Given that the primary benefit of invasive treatment for obstructive coronary artery disease is relief of symptoms, we think that the threshold for invasive angiography may need to be higher in asymptomatic patients, for whom the potential benefits remain uncertain.

Other areas of potential improvement include risk stratification and the quality of noninvasive testing. Neither the Framingham risk score in the case of asymptomatic patients nor the Diamond and Forrester method in the case of symptomatic patients takes into account certain clinical risk factors, such as peripheral vascular disease, that are known to be important in this cohort. Even with the lack of certainty regarding the range of tests included in the NCDR, the incremental information provided by the aggregate of all forms of noninvasive testing was limited. Hence, our data support ongoing efforts to im- prove overall strategies for patient selection, including, but not limited to, improving the quality of noninvasive testing, in order to determine the optimal decision-making algorithms for the evaluation of suspected obstructive coronary artery disease.

The CathPCI Registry of the NCDR is a large, national registry that represents contemporary clinical practice in the community. Building on these strengths, our analysis provides current information on the way in which the entire diagnostic process - including assessment of clinical factors, symptoms, and noninvasive testing - functions in the referral of patients for diagnostic catheterization.

Our study has several limitations. We could not distinguish between patients who underwent resting electrocardiography, echocardiography, or CT and those who underwent stress testing, and we do not have any information regarding the undoubtedly large population of patients who were evaluated but did not undergo cardiac catheterization. In addition, the analysis of angiographic findings is limited by the fact that the assessment of coronary stenosis was made by the interpreting physician. Finally, the performance of the Framingham risk score may be underestimated because we imputed values for lipid levels and blood pressure.

In summary, in a large, national registry, only $38 \%$ of patients without known heart disease who underwent elective invasive angiography had obstructive coronary artery disease. Current strategies that are used to inform decisions regarding invasive angiography, including clinical assessment of risk and noninvasive testing, need to be improved substantially to increase the diagnostic yield of cardiac catheterization in routine clinical practice.

\footnotetext{
Supported by unrestricted funding from the American College of Cardiology's National Cardiovascular Data Registry CathPCI Registry.

Dr. Patel reports serving on the paid advisory board on Fabry's disease for Genzyme; Dr. Peterson, receiving grant support from Bristol-Myers Squibb, Sanofi-Aventis, and Schering-Plough (now Merck); Dr. Brennan, receiving lecture fees from Daiichi-Sankyo; and Dr. Anderson, receiving consulting fees from Premier, lecture fees from Sanofi-Aventis and Bristol-Myers Squibb, and grant support from AstraZeneca. No other potential conflict of interest relevant to this article was reported.
} 
REFERENCES

1. Cheitlin MD, Armstrong WF, Aurigemma GP, et al. ACC/AHA/ASE 2003 guideline update for the clinical application of echocardiography: summary article: a report of the American College of Cardiology/American Heart Association Task Force on Practice Guidelines (ACC) AHA/ASE Committee to Update the 1997 Guidelines for the Clinical Application of Echocardiography). J Am Soc Echocardiogr 2003;16:1091-110.

2. Klocke FJ, Baird MG, Lorell BH, et al. ACC/AHA/ASNC guidelines for the clinical use of cardiac radionuclide imaging - executive summary: a report of the American College of Cardiology/American Heart Association Task Force on Practice Guidelines (ACC/AHA/ASNC Committee to Revise the 1995 Guidelines for the Clinical Use of Cardiac Radionuclide Imaging). J Am Coll Cardiol 2003;42:131833.

3. Fraker TD Jr, Fihn SD. 2007 Chronic angina focused update of the ACC/AHA 2002 guidelines for the management of patients with chronic stable angina: a report of the American College of Cardiology/American Heart Association Task Force on Practice Guidelines Writing Group to develop the focused update of the 2002 guidelines for the management of patients with chronic stable angina. J Am Coll Cardiol 2007;50:2264-74.

4. Lucas FL, DeLorenzo MA, Siewers AE, Wennberg DE. Temporal trends in the utilization of diagnostic testing and treatments for cardiovascular disease in the
United States, 1993-2001. Circulation 2006; 113:374-9.

5. Weintraub WS, McKay CR, Riner RN, et al. The American College of Cardiology National Database: progress and challenges. J Am Coll Cardiol 1997;29:459-65.

6. Brindis RG, Fitzgerald S, Anderson HV, Shaw RE, Weintraub WS, Williams JF. The American College of Cardiology-National Cardiovascular Data Registry (ACC-NCDR): building a national clinical data repository. J Am Coll Cardiol 2001;37:2240-5.

7. Zeger SL, Liang KY, Albert PS. Models for longitudinal data: a generalized estimating equation approach. Biometrics 1988;44:1049-60. [Erratum, Biometrics 1989;45:347.]

8. DeLong ER, DeLong DM, Clarke-Pearson DL. Comparing the areas under two or more correlated receiver operating characteristic curves: a nonparametric approach. Biometrics 1988;44:837-45.

9. Bashore TM, Bates ER, Berger PB, et al. American College of Cardiology/Society for Cardiac Angiography and Interventions Clinical Expert Consensus Document on cardiac catheterization laboratory standards: a report of the American College of Cardiology Task Force on Clinical Expert Consensus Documents. J Am Coll Cardiol 2001;37:2170-214

10. Bernstein SJ, Laouri M, Hilborne LH, et al. Coronary angiography: a literature review and ratings of appropriateness and necessity. Santa Monica, CA: RAND, 1992. 11. Kemp HG, Kronmal RA, Vlietstra RE, Frye RL. Seven year survival of patients with normal or near normal coronary arteriograms: a CASS registry study. J Am Coll Cardiol 1986;7:479-83.

12. Johnson LW, Lozner EC, Johnson S, et al. Coronary arteriography 1984-1987: a report of the Registry of the Society for Cardiac Angiography and Interventions. I. Results and complications. Cathet Cardiovasc Diagn 1989;17:5-10.

13. Johnson LW, Krone R. Cardiac catheterization 1991: a report of the Registry of the Society for Cardiac Angiography and Interventions (SCA\&I). Cathet Cardiovasc Diagn 1993;28:219-20.

14. Shaw LJ, Shaw RE, Merz CNB, et al. Impact of ethnicity and gender differences on angiographic coronary artery disease prevalence and in-hospital mortality in the American College of Cardiology-National Cardiovascular Data Registry. Circulation 2008;117:1787-801.

15. Diamond GA, Forrester JS. Analysis of probability as an aid in the clinical diagnosis of coronary-artery disease. $\mathrm{N}$ Engl J Med 1979;300:1350-8.

16. Chaitman BR, Bourassa MG, Davis K, et al. Angiographic prevalence of highrisk coronary artery disease in patient subsets (CASS). Circulation 1981;64:360-7.

17. Lin GA, Dudley RA, Lucas FL, Malenka DJ, Vittinghoff E, Redberg RF. Frequency of stress testing to document ischemia prior to elective percutaneous coronary intervention. JAMA 2008;300:1765-73.

Copyright ( 2010 Massachusetts Medical Society. only if they have been registered (see N Engl J Med 2004;351:1250-1).

Current information on requirements and appropriate registries is available at www.icmje.org/faq.pdf. 